\title{
Reducing Transmission Losses in Hollow THz Waveguides
}

\author{
Oleg Mitrofanov, Richard James, F. Aníbal Fernández, Member, IEEE, Themistoklis K. Mavrogordatos, and \\ James A. Harrington
}

(Invited Paper)

\begin{abstract}
Research on reducing material absorption in Terahertz (THz) waveguides has lead to development of guiding structures with transmission losses as low as $1 \mathrm{~dB} / \mathrm{m}$. Among waveguides that exhibit low loss at $\mathrm{THz}$ frequencies are the dielectriclined hollow cylindrical metallic waveguides. Loss reduction in this waveguide is attributed to an ideal profile of the dominant hybrid $\mathrm{HE}_{11}$ mode. This mode profile also results in relatively low dispersion and very high coupling efficiency. In this contribution we overview properties of dielectric-lined hollow cylindrical metallic waveguides for $\mathrm{THz}$ waves, their design principles and the fabrication process. The impact of the mode profile on losses and dispersion at $\mathrm{THz}$ frequencies is confirmed experimentally by $\mathrm{THz}$ near-field imaging and THz time-domain spectroscopy and numerically by the finite element method.
\end{abstract}

Index Terms-Dispersion, near-field microscopy, terahertz waveguides, $\mathrm{THz}$ spectroscopy, transmission loss, waveguide modes.

\section{INTRODUCTION}

G UIDED terahertz $(\mathrm{THz})$ wave propagation is necessary for a range of applications in $\mathrm{THz}$ systems, from simple power delivery to sensitivity enhancing in spectroscopy and resolution improving in imaging. Early studies of $\mathrm{THz}$ waveguides were adapting existing designs that had already been developed for optical waves and radiowaves [1]-[6]. However, the most prominent solutions, the dielectric cylindrical fiber for near-infrared and optical waves and the coaxial metallic waveguide for radiowaves, both yield large transmission losses at $\mathrm{THz}$ frequencies [2], [5]. The poor performance is caused by the increase of Ohmic losses in metals at $\mathrm{THz}$ frequencies and the lack of sufficiently transparent dielectric materials. The challenge of mitigating these losses requires alternative solutions and it has

Manuscript received March 15, 2011; revised April 30, 2011; accepted May 10, 2011. Date of current version August 31, 2011. This work was supported in part by the Royal Society under Grant UF080745 and the Engineering and Physical Sciences Research Council under Grant EP/G033870/1.

O. Mitrofanov and F. A. Fernandez are with the University College London, London, WC1E 7JE, U.K. (e-mail: o.mitrofanov@ucl.ac.uk).

R. James is with the University College London, London, WC1E 7JE, U.K., and also with Ghent University, 9000 Gent, Belgium.

T. K. Mavrogordatos is with Cambridge University, Cambridge, CB2 1PZ, U.K..

J. A. Harrington is with Rutgers University, Piscataway, NJ 08854 USA.

Color versions of one or more of the figures in this paper are available online at http://ieeexplore.ieee.org.

Digital Object Identifier 10.1109/TTHZ.2011.2159547 transformed studies of $\mathrm{THz}$ waveguides into an active research area.

Development of $\mathrm{THz}$ waveguides with reduced losses has seen a rapid progress in the recent years: the attenuation level has been decreased from over $100 \mathrm{~dB} / \mathrm{m}$ to approximately 1 $\mathrm{dB} / \mathrm{m}[6]-[17]$, comparable to transmitting $\mathrm{THz}$ waves in air. The low-loss characteristics so far have been achieved only in structures, which guide $\mathrm{THz}$ waves within a large hollow or porous core. Among designs with the lowest losses are the dielectric-lined hollow cylindrical metallic waveguides, which will be in the focus of this article.

This waveguide is a small modification of the hollow metallic waveguide, which nevertheless makes a significant impact on the waveguide characteristics at $\mathrm{THz}$ frequencies: (1) transmission losses can be reduced from about $3-5 \mathrm{~dB} / \mathrm{m}$ in the metallic guides [6] to the level below $1 \mathrm{~dB} / \mathrm{m}$ [16], [17]; (2) the waveguide exhibits small dispersion, practically independent of the dielectric material used for the coating [18]; and (3) the dominant mode profile allows very efficient coupling to free space $\mathrm{THz}$ beams [19], [20]. These properties make the dielectriclined hollow cylindrical metallic waveguides attractive for a range of $\mathrm{THz}$ applications.

Most low-loss THz waveguides use large cores and therefore support multiple modes of wave propagation. As losses vary significantly for different modes, the impact of mode profile on losses and dispersion became central in studies of $\mathrm{THz}$ waveguides [19]-[21]. To enable investigation of waveguide modes, specialized characterization methods have been developed. In particular, THz time-domain spectroscopy (THz-TDS), which has been used widely for characterization of waveguide losses and dispersion, was recently applied for visualization of the fields propagating in waveguides [10], [20]. It provided a possibility to determine waveguide normal modes, mode composition, and mode-specific dispersion characteristics [18].

Reducing transmission losses is not always the main goal in designing waveguides. Some applications of waveguides make it difficult to avoid losses. For example, waveguides for sensing or for 'on-chip' signal transmission require confinement of the traveling wave, often to the subwavelength level; in such waveguides, transmitted energy is intentionally concentrated near metallic interfaces, causing large losses. Therefore, among many noteworthy $\mathrm{THz}$ waveguides that have been investigated recently [1]-[30], only a few designs exhibit low transmission loss. In plasmonic metallic waveguides, for example, the loss could be as high as $100 \mathrm{~dB} / \mathrm{m}$ at THz frequencies [29]. 


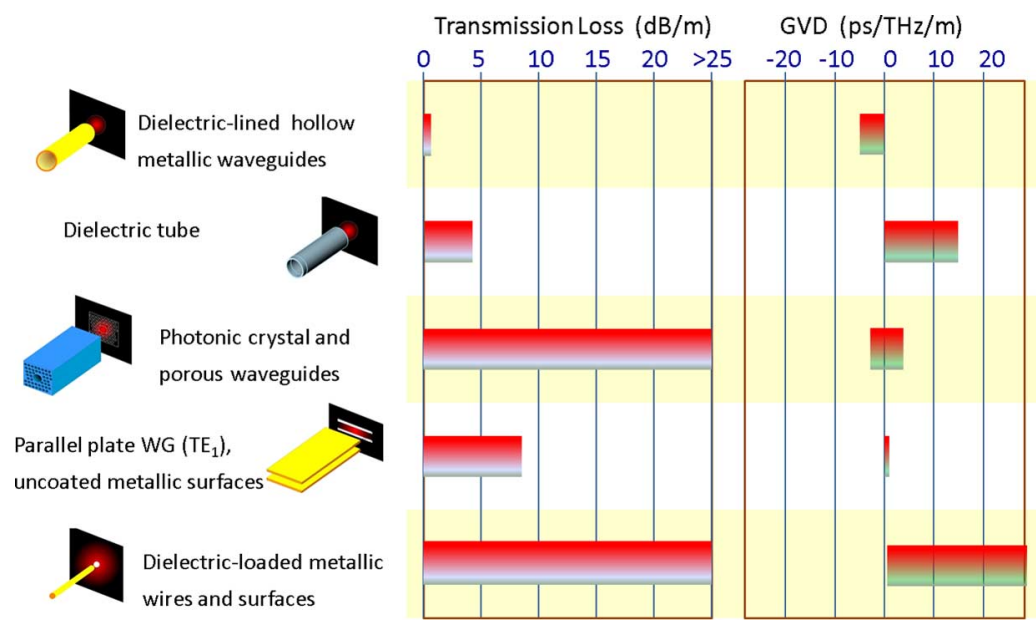

Fig. 1. Common THz waveguides and their estimated transmission loss and dispersion characteristics in the region of 1-2 THz [7]-[23].

Here we will discuss only low-loss waveguides and focus mainly on the dielectric-lined hollow cylindrical metallic waveguides as an example in which elimination of material absorption is achieved to a high extent. We will overview the general design principle, the fabrication process and results of experimental investigations and numerical modeling.

\section{LOW-LOSS THz WAVEGUIDES}

Transmission losses in $\mathrm{THz}$ waveguides primarily arise from two sources: the Ohmic losses on metallic surfaces and absorption inside dielectrics. At optical frequencies there are dielectric materials with extremely small absorption and at sub- $\mathrm{THz}$ frequencies the Ohmic losses are relatively low. At THz frequencies however neither metallic waveguides nor dielectric waveguides offer a clear advantage over each other.

Minimizing the impact of material losses leads to one general principle for all low-loss $\mathrm{THz}$ waveguides: the transmitted energy must be distributed in the hollow region of the waveguide. Most waveguide designs that have been considered for reducing material losses follow this principle. These waveguides can be divided into three categories: hollow metallic waveguides, photonic crystal waveguides, and dielectric porous and pipe waveguides. Reported losses and dispersion for waveguides in these categories are summarized in Fig. 1.

To reduce losses in metallic waveguides, the amount of transmitted energy near metallic surfaces of the waveguide must be minimized. The waveguide mode profile therefore directly affects the transmission loss characteristics. It is useful to consider the parallel plate waveguide (PPWG) to illustrate the impact of the mode profile. The transverse electromagnetic (TEM) mode of the PPWG exhibits a uniform distribution of the electric field between the waveguide plates, whereas the electric field of the transverse electric $\left(\mathrm{TE}_{1}\right)$ mode is vanishing on the metallic surfaces. As a result, the Ohmic losses are substantially higher for the TEM mode compared to the $\mathrm{TE}_{1}$ mode. The TEM mode losses increase for higher frequencies and for smaller plate separation. The losses for the $\mathrm{TE}_{1}$ mode however decrease with frequency for sufficiently large plate separation $d \gg \lambda$ [23].

The behavior of the TEM and $\mathrm{TE}_{1}$ modes in the PPWG illustrates the difference in absorption for two mode types in metallic waveguides, with the electric field vector either normal or tangential to the metallic surface. In hollow cylindrical metallic waveguides, the $\mathrm{TM}_{01}$ and $\mathrm{TE}_{01}$ modes exhibit similar behavior to the TEM and $\mathrm{TE}_{1}$ modes of the PPWG respectively. Similar to the $\mathrm{TE}_{1}$ mode in the PPWG, the $\mathrm{TE}_{01}$ mode is the lowest loss mode for the hollow cylindrical metallic waveguide at $\mathrm{THz}$ frequencies [19], [20]: we measured loss of $\sim 3 \mathrm{~dB} / \mathrm{m}$ at $2.5 \mathrm{THz}$ in a $2.2 \mathrm{~mm}$ bore Ag-coated waveguide for the $\mathrm{TE}_{01}$ mode [6], [19]. Modes with the electric field vector normal to the surface, such as the $\mathrm{TM}_{01}$ mode in the cylindrical waveguide, generally experience much higher Ohmic losses. The more commonly used $\mathrm{TE}_{11}$ mode contains both the tangential and the normal components near the metallic surface. This mode exhibits intermediate loss figures: in a $3 \mathrm{~mm}$ bore copper waveguide we measured loss of $3.9 \mathrm{~dB} / \mathrm{m}$ at $1.89 \mathrm{THz}[6]$.

It is important to realize that minimizing losses in hollow core $\mathrm{THz}$ waveguides inevitably leads to designs where the core is much larger than the wavelength. For example, transmission losses in the hollow cylindrical metallic waveguide are inversely proportional to the third power of the waveguide diameter [31]. Most low-loss $\mathrm{THz}$ waveguides, as a result, support several modes of wave propagation.

The other two categories of low-loss $\mathrm{THz}$ waveguides are purely dielectric waveguides. In both the photonic crystal waveguides and the porous waveguides, the core is kept predominantly hollow to reduce absorption by the dielectric materials. The guiding mechanism in the photonic crystal waveguides is based on multiple reflections experienced by the travelling wave in the cladding. In the porous waveguides, the guiding mechanism is based on minimizing the overlap between the core mode and the cladding modes. Although most of the energy is propagating in the hollow regions, absorption inside the dielectric regions is still limiting the overall losses. A recently demonstrated photonic crystal waveguide made of Topas, a polymer material with relatively low absorption coefficient of $1 \mathrm{~dB} / \mathrm{cm}$, exhibits the overall loss of $20-40 \mathrm{~dB} / \mathrm{m}$ [10]. The pipe waveguide (dielectric tube) is another simple implementation of the air core concept. The traveling wave, weakly confined by reflections in the dielectric walls, experiences the overall loss of $2 \mathrm{~dB} / \mathrm{m}$ at $0.3-0.4 \mathrm{THz}$ [8] and 
$\sim 4 \mathrm{~dB} / \mathrm{m}$ at $1 \mathrm{THz}$ [7]. To reduce losses in these waveguides further, dielectric materials with lower absorption at $\mathrm{THz}$ frequencies are required. High-resistivity silicon is one of potential candidates: the upper limit for the intrinsic absorption coefficient is less then $0.01 \mathrm{~cm}^{-1}$, corresponding to the loss of less then $4.3 \mathrm{~dB} / \mathrm{m}$, over the range of $0.2-1 \mathrm{THz}$ [32]. A solid core waveguide made of high-resistivity silicon can potentially provide low-loss transmission in the single-mode regime.

The waveguide that will be discussed in the following sections, the dielectric-lined hollow cylindrical metallic waveguide, allows reducing the Ohmic losses along the entire circumference of the waveguide walls for a linearly polarized wave. This is achieved by adding a thin dielectric layer on the inner metallic surface. In contrast to the hollow metallic waveguides with losses of $\sim 3-5 \mathrm{~dB} / \mathrm{m}$, a dielectric-lined waveguide exhibits a measured loss of $0.95 \mathrm{~dB} / \mathrm{m}$ at $2.5 \mathrm{THz}$ [16]. The loss reduction can be explained by the ideal profile of the dominant waveguide mode, the hybrid $\mathrm{HE}_{11}$ mode. This mode has the minimal electric field amplitude near the walls, similar to the $\mathrm{TE}_{01}$ mode in the cylindrical metallic waveguide and to the $\mathrm{TE}_{1}$ mode in the PPWG, and therefore it also experiences small absorption. In addition, the $\mathrm{HE}_{11}$ mode is completely confined in the core of the waveguide, unlike the $\mathrm{TE}_{1}$ mode in the PPWG, and it is linearly polarized, unlike the $\mathrm{TE}_{01}$ mode. These properties make the dielectric-lined hollow cylindrical metallic waveguides ideal for coupling to free space propagating beams and useful for practical applications.

\section{Dielectric-Lined Hollow Metallic WaVeguides}

The dielectric-lined hollow cylindrical metallic waveguide is a modified hollow metallic waveguide, where the inner metallic surface is coated with a thin dielectric layer (typically 10-20 microns). The modified structure changes the profile of the dominant mode, the $\mathrm{HE}_{11}$ mode, in a way that the transmitted energy is concentrated near the waveguide center. This mode has substantially smaller penetration into the absorbing metallic wall compared to any other mode of the metallic guide and it implements the principle of guiding the waves in the hollow core to the highest extent.

Unlike the classical TE and TM modes in metallic waveguides, the profile of the hybrid mode is wavelength dependent. Analytical treatment of the waveguide loss and dispersion characteristics is therefore somewhat difficult and no exact mathematical expression exist [33]-[35]. The waveguide properties were therefore evaluated experimentally [16]-[20] and numerically [36], [37]. Despite the mode profile dependency on the wavelength, the waveguide exhibits low loss and relatively small dispersion in a wide band of frequencies. In the waveguides designed for $2.5 \mathrm{THz}$, we observed transmission losses around $1 \mathrm{~dB} / \mathrm{m}$ and dispersion of $6 \mathrm{ps} / \mathrm{THz} / \mathrm{m}$ within a bandwidth of at least $1 \mathrm{THz}$ [16], [18].

The overall dispersion is primarily defined by the radius of the waveguide and it is practically independent of the dielectric coating dispersion [18]. For the $\mathrm{HE}_{11}$ mode, the dielectric coating has the opposite effect to the surface wave collapse experienced by waves propagating on dielectric-coated metallic wires and planes, where the coating attracts the wave energy closer to the metallic surface and causes large transmission losses and dispersion (Fig. 1) [21], [22]. In the dielectric-lined hollow waveguides, the energy can be pushed away from the surface, resulting in minimal losses and dispersion.

This combination of low loss and low dispersion is unique for $\mathrm{THz}$ waveguides. The width of the low-loss region is sufficiently large to allow transmission of broadband $\mathrm{THz}$ pulses [18]. It is important to note however that the ideal mode profile is formed only if the core diameter is larger than the wavelength [33], [35]. These waveguides therefore also support higher order modes.

Research on dielectric-lined waveguides for $\mathrm{THz}$ waves have experienced much activity in the last several years with a focus on realization of good quality guides, modeling, and on integration of waveguides with $\mathrm{THz}$ devices [16]-[20], [36]-[39]. Modifications of the waveguide walls have also been recently considered with the aim to reduce transmission losses even further [9]. The original studies of this waveguide type however date back to the 1970 s, when the dielectric-lined waveguides were considered for communication systems prior to the widespread use of the silica glass optical fibers [33]-[35]. In the THz and mid-infrared regions, sufficiently transparent dielectric materials have not been found yet and the dielectric-lined cylindrical metallic waveguides currently provide superior characteristics (Fig. 1).

\section{WaVEguide FABRICATION Methods}

In this section we discuss fabrication of hollow waveguides made from glass or polymer tubing. Some of our earlier work involved the deposition of a thin dielectric film of AgI over the Ag layer to lower the waveguide losses in the infrared (IR) [31]. A significant challenge in extending the metal/dielectric, hollow waveguide technology from IR wavelengths to $\mathrm{THz}$ frequencies is that a much thicker dielectric layer is required at $\mathrm{THz}$ frequencies. This may be seen from (1), which shows that the optimum dielectric layer thickness, $d_{d}$, is proportional to the design wavelength, $\lambda_{0}$ :

$$
d_{d}=\frac{\lambda_{0}}{2 \pi \sqrt{n_{d}^{2}-1}} \tan ^{-1}\left[\frac{n_{d}}{\left(n_{d}^{2}-1\right)^{1 / 4}}\right]
$$

where $n_{d}$ is the refractive index of the dielectric [31]. This expression is derived with the assumption that there is no absorption inside the dielectric layer. The optimal thickness is slightly smaller for absorptive films [36]. The wavelength of THz radiation is 10 to 100 times longer than IR radiation; hence the dielectric film thickness of a metal/dielectric hollow core waveguide designed for $\mathrm{THz}$ frequency radiation must be 10 to 100 times greater than those designed for IR radiation.

We have chosen to use a polymer film instead of $\mathrm{AgI}$ as the dielectric layer. In general there are several advantages to the use of polymer films. Firstly, they are relatively transparent in the $\mathrm{THz}$ region [36] and secondly it is not difficult to deposit relatively thick and smooth coatings using liquid-phase chemistry methods [38]. We have chosen to deposit polystyrene (PS) because it easily dissolves in toluene.

The first step in the preparation of the $\mathrm{Ag} / \mathrm{PS}$ waveguides is to deposit an $\mathrm{Ag}$ film [31] within a glass substrate tube by 


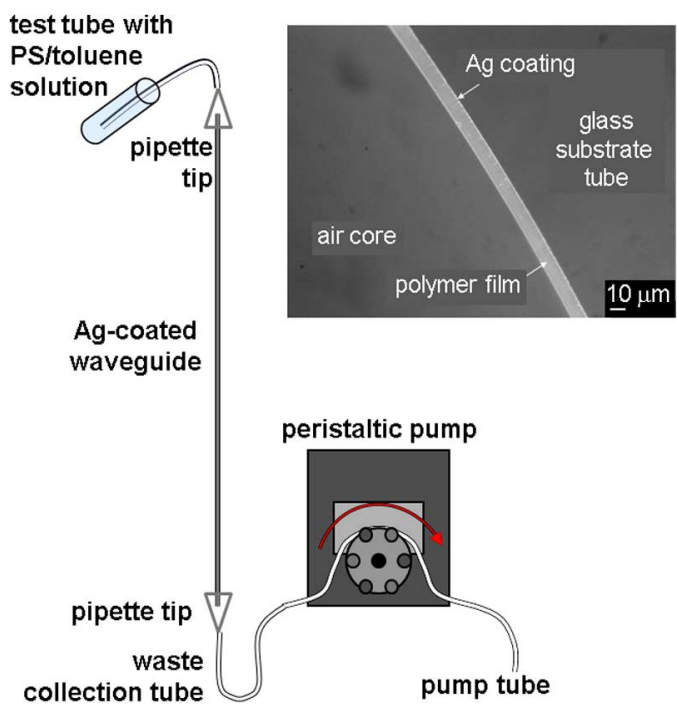

Fig. 2. A schematic diagram of the apparatus used to deposit polymer thin films over Ag films. Inset: an optical micrograph of a $2.2 \mathrm{~mm}$ bore diameter $\mathrm{Ag} / \mathrm{PS}$ waveguide showing the PS layer [38].

combining a solution containing $\mathrm{AgNO}_{3}$ and $\mathrm{NH}_{4} \mathrm{OH}$ with a reducing solution containing dextrose and $\mathrm{Na}_{2}$ EDTA. The Ag film grows at a rate of approximately $1 \mu \mathrm{m} / \mathrm{hr}$ and our Ag films were normally about $1 \mu \mathrm{m}$ thick. This is much thicker than the skin depth for $\mathrm{Ag}$ at $\mathrm{THz}$ frequencies $(\sim 100 \mathrm{~nm}$ at $2.5 \mathrm{THz})$ and, therefore, no $\mathrm{THz}$ radiation can penetrate the waveguide wall. Rigid glass tubing with bore sizes ranging between 1.6 and $2.2 \mathrm{~mm}$ were used to fabricate $120 \mathrm{~cm}$ long waveguides.

The PS films were deposited over the Ag coatings using a liquid-flow coating process similar to Abe et al. [40] in which a PS/toluene solution is drawn through the waveguide at a constant coating velocity using a peristaltic pump. A schematic diagram of the coating apparatus is shown in Fig. 2. The system uses a Masterflex L/S peristaltic pump configured with a microbore tubing pump head. The bottom opening is connected to a U-shaped Nalgene waste tube that collects the excess solution as it exits the waveguide. Some pulsation of the volume flow rate is inherent in systems utilizing a peristaltic pump, thus the coating velocity is also pulsed. The use of a microbore pump tube minimizes the amplitude of the pulsation. Once the pump produces a $10 \mathrm{~cm}$ column of solution in the tubing, the source tube is disconnected. This column travels along the length of the waveguide producing a thin film of PS/toluene solution over the Ag coating. After the coating material passes through the tubing the waveguide is dried by forcing air through its core at a rate of $1 \mathrm{~L} / \mathrm{min}$.

The inset in Fig. 2 shows an optical micrograph of the cross-section of a $2.2 \mathrm{~mm}$ bore diameter $\mathrm{Ag} / \mathrm{PS}$ hollow waveguide. A $25 \mathrm{wt} . \% \mathrm{PS} /$ toluene solution and a coating velocity of $4.5 \mathrm{~cm} / \mathrm{min}$ were used to deposit the PS film. The PS film is uniform around the circumference of the waveguide and its thickness is about $11 \mu \mathrm{m}$. The film and Ag coating adhere well to each other and the glass substrate tube. The surface of the PS film is relatively smooth and it compares well with the AgI dielectric films deposited over Ag for the IR region. PS films ranging from $1 \mu \mathrm{m}$ to $17 \mu \mathrm{m}$ in thickness have been deposited with this method by varying the PS concentration and the flow rate [38]. Waveguides discussed here were fabricated using rigid glass tubing, however flexible waveguides have also been made from $1 \mathrm{~mm}$ bore polycarbonate tubing.

\section{Characterization of WaVeguides at THz Frequencies}

The choice of characterization methods at $\mathrm{THz}$ frequencies is gradually improving, however it is nevertheless limited. Terahertz time-domain spectroscopy (THz-TDS) has been extremely useful for waveguide characterization because it provides the ability to measure loss and dispersion spectra in a broad range of frequencies. The multimode nature of most low-loss $\mathrm{THz}$ waveguides however requires that the characterization method differentiates between waveguide modes. To develop this capability, we combined THz-TDS with THz near-field microscopy. These techniques allowed us to map propagating waves at the waveguide output in space and time and consequently determine which modes travel in the waveguide [20]. The knowledge of mode composition removes any ambiguity in analysis of the THz-TDS spectra, it allowed us to measure mode-specific dispersion characteristics for the dielectric-lined hollow metallic waveguides.

\section{A. THz Time-Domain Spectroscopy and Space-Time Mode Mapping}

In the THz-TDS application to waveguide characterization, a THz pulse is sent through the waveguide and the transmission loss and dispersion characteristics are determined directly from the Fourier spectra of the $\mathrm{THz}$ pulse waveforms before and after transmission through the waveguide. There are two difficulties with applying the standard THz-TDS approach. First of all, the $\mathrm{THz}$ pulse can travel through the waveguide as a superposition of several modes and therefore the spectrum may be affected by interference of these modes. The mode interference produces a periodic series of maxima and minima in the waveguide transmission spectrum measured by THz-TDS. Secondly, coupling of $\mathrm{THz}$ waves in and out of the waveguides is typically frequency dependent and the coupling efficiency for all frequency components of the $\mathrm{THz}$ pulse must be taken into account. The waveguide characterization therefore becomes a two-fold problem, where mode composition must be determined prior the measurement of dispersion properties.

To avoid the uncertainties related to the multimode propagation and the frequency dependent coupling, the mode composition is determined experimentally. A schematic diagram of the measurement system is shown in Fig. 3(a). The first waveguide (wg 1) serves to define modes that are launched into the test waveguide (wg 2). As the $\mathrm{THz}$ pulse enters the launch waveguide, its energy splits between waveguide modes in a way that their superposition matches the incident beam profile. The excited modes then travel through the waveguide with their unique group velocities. After propagation through the launch waveguide, the modes become separated in time.

The corresponding space-time distribution of the $\mathrm{THz}$ pulse field is detected by a high-resolution near-field $\mathrm{THz}$ probe at the waveguide output end [20]. The time dependence is recorded using a variable time-delay stage and the spatial dependence is 
(a)

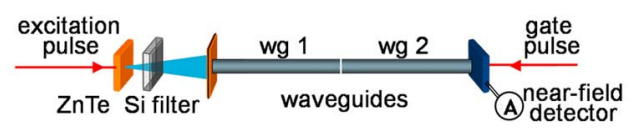

(b)

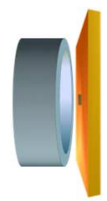

(c)
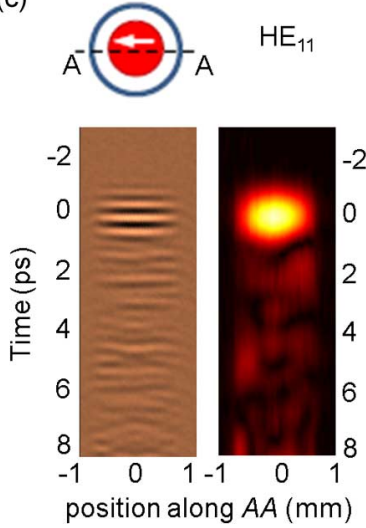

(d)
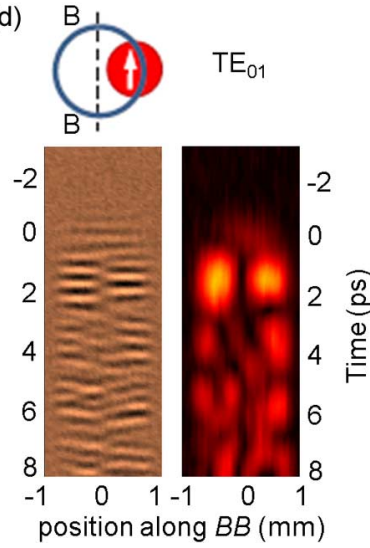

Fig. 3. (a) A schematic diagram of the experimental system for near-field mapping of waveguide modes. (b) A close-up of the waveguide output and the near-field field probe. (c) Space-time maps of the horizontal component of the electric field $\left(E_{x}\right)$ for the $\mathrm{HE}_{11}$ mode at the output end of a $1.8 \mathrm{~mm}$ diameter $133.5 \mathrm{~mm}$ long $\mathrm{Ag} / \mathrm{PS}$ waveguide: the left panel shows the measured electric field and the right panel shows the amplitude of the $2.3 \mathrm{THz}$ component, near the pulse spectrum maximum. The $\mathrm{HE}_{11}$ mode is excited selectively by placing a $1 \mathrm{~mm}$ diameter pinhole at the waveguide entrance as shown in the schematic diagram. (d) Space-time maps and the excitation arrangement for the $\mathrm{TE}_{01}$ mode in the same waveguide.

recorded by scanning the output end of the waveguide with respect to the near-field probe [Fig. 3(b)] along the waveguide diameter [18]. The output wave can be displayed as a space-time map, where the excited modes can be identified as regions of strong electric field. Relative amplitudes of the excited modes can be estimated by calculating the spectral amplitude distribution directly from the data using the local Fourier transform with a 2-ps time window [20].

Fig. 3(c), (d) shows the measured space-time maps for a $1.8 \mathrm{~mm}$ bore diameter $133.5 \mathrm{~mm}$ long Ag/PS waveguide and two different coupling conditions, which result in excitation of the two lowest loss modes, the linearly polarized $\mathrm{HE}_{11}$ mode and the azimuthally polarized $\mathrm{TE}_{01}$ mode. These modes are excited by controlling the input field pattern with a $1 \mathrm{~mm}$ diameter pinhole. The $\mathrm{HE}_{11}$ mode is launched through the input pinhole centered on the waveguide axis [Fig. 3(c), schematic diagram]. The $\mathrm{TE}_{01}$ mode, which is difficult to detect by the standard THz-TDS system because the mode symmetry inhibits coupling to a far-field dipole antenna detector, is excited by shifting the pinhole off the waveguide axis in the direction perpendicular to the electric field polarization as shown in Fig. 3(d).

The space-time maps at the output of the launch waveguide display the composition of modes entering the test waveguide. This mode composition is then compared to the mode composition at the test waveguide output end obtained from another space-time map. These data provide all required information to determine loss and dispersion in the test waveguide. The imaging system also provides a means for waveguide alignment and for selective mode excitation.
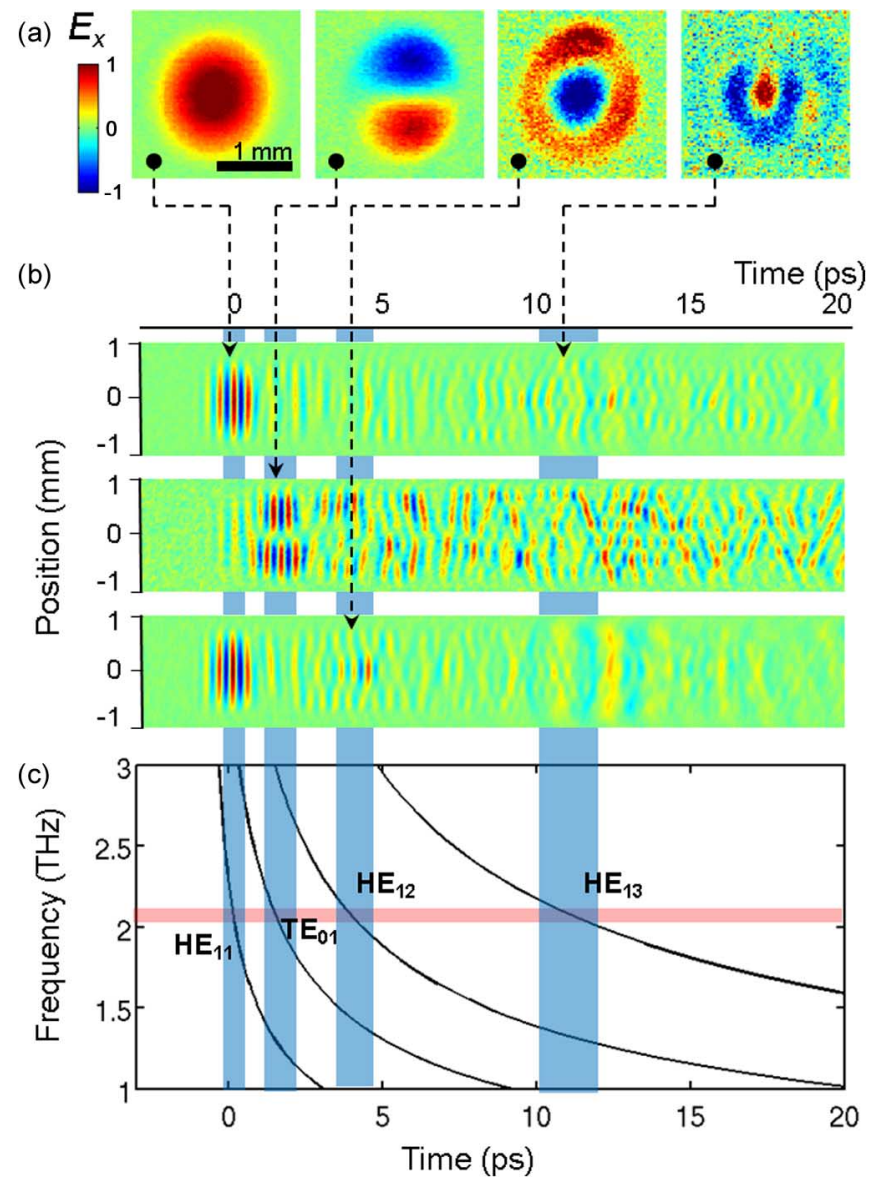

Fig. 4. Time-resolved near-field mode mapping. (a) Two-dimensional distributions of the electric field component $E_{x}(x, y)$ are shown in the top row for various time delays corresponding to the arrival of different modes [18]. (b) Space-time maps at the output of the $133.5 \mathrm{~mm}$ long $\mathrm{Ag} / \mathrm{PS}$ waveguide for three different coupling configurations: through a $1 \mathrm{~mm}$ diameter pinhole centered on the waveguide axis (top map); through the pinhole positioned off-axis as in Fig. 3 (middle map); and the uniform waveguide input illumination (bottom map). (c) Estimated temporal delay for higher order modes. The origin of the time axis $t=0$ is chosen to mark the arrival of the dominant $\mathrm{HE}_{11}$ mode.

\section{B. Time-Resolved Near-Field Mode Imaging}

The same near-field probe system [Fig. 3(a)] can be used to determine spatial profiles of normal waveguide modes. The measured maps show that the individual modes become separated in time after traveling in the waveguide due to each mode having a unique group velocity. The spatial mode profile for a selected mode therefore can be measured at the moment when this mode reaches the detector, using the time-resolved capability of the THz-TDS system.

Fig. 4(a) shows two-dimensional spatial maps of four normal modes in the dielectric-lined hollow cylindrical metallic waveguide detected at the waveguide output by the near-field detector. The space-time maps in Fig. 4(b) show that the corresponding modes are not overlapping in time and therefore the profiles are not affected by mode interference. Fig. 4(c) shows estimated temporal delay after propagation in the $133.5 \mathrm{~mm}$ long waveguide for the modes in Fig. 4(a). The time delay is calculated from the group velocity $v_{g}=d \omega / d k$ analytically, assuming that the waveguide dispersion is described by the cut-off frequencies of $0.128,0.203,0.293$ and $0.459 \mathrm{THz}$ for $\mathrm{HE}_{11}$, 
$\mathrm{TE}_{01}, \mathrm{HE}_{12}$ and $\mathrm{HE}_{13}$ modes respectively [18]. Fig. 4(c) illustrates that for the $\mathrm{THz}$ pulse with the center frequency of $2.1 \mathrm{THz}$, the $\mathrm{TE}_{01}, \mathrm{HE}_{12}$ and $\mathrm{HE}_{13}$ modes are delayed by approximately 2,4 , and 11 ps compared to the dominant $\mathrm{HE}_{11}$ mode.

The time-resolved mode profile mapping is quite different from the standard THz-TDS approach, where complete waveforms are collected for every point of the two-dimensional spatial map. The electric field amplitude is then calculated for desired frequencies using the Fourier transform. The standard approach works well if only a single mode propagates in the waveguide. The measured maps in this case show the mode profile. However, if multiple modes are present, the standard method suffers from mode interference [20] and measured profiles display interference patters rather than the individual modes.

The time-resolved mode profile mapping on the other hand can be applied for multimode propagation because different modes reach the near-field detector at different times. It is critical however that the waveguide length is long enough for the modes to acquire a sufficient time delay. This method provided the direct experimental confirmation that the dielectric layer transforms the dominant mode of the metallic $\mathrm{THz}$ waveguide into the low-loss hybrid $\mathrm{HE}_{11}$ mode [20]. In addition, it enabled experimental characterization of other modes, including the $\mathrm{TE}_{01}$ mode, which requires special coupling conditions [18].

\section{Waveguide Loss and Dispersion Measurements}

Dispersion characteristics of individual modes can be measured using THz-TDS after the mode structure and composition at the output of the launch waveguide have been determined. To minimize spectral artifacts caused by the mode interference, the near-field probe can be positioned in a region of the mode profile maximum or in a location where the second strongest mode has a minimum. Coupling of the $\mathrm{THz}$ beam into the launch waveguide can also be adjusted in a way that the mode of interest is the strongest. This approach was applied to find dispersion characteristics of the dominant $\mathrm{HE}_{11}$ mode as well as modes that exhibit low coupling efficiencies, such as the $\mathrm{TE}_{01}$ mode [18]. The $\mathrm{HE}_{11}$ dispersion characteristics will be discussed in the next section together with results of numerical modeling.

Experimental characterization of the transmission loss spectrum is a more difficult task, particularly if the overall signal attenuation in a waveguide sample is only a fraction of $1 \mathrm{~dB}$. Multimode interference in such cases can produce large systematic errors in characteristics measured by THz-TDS. Fig. 5(a) shows typical examples of spectra measured by THz-TDS at the output of a 15 and $30 \mathrm{~cm}$ long waveguide samples. Ideally, the difference in the measured spectra represents the transmission loss per $15 \mathrm{~cm}$ of the waveguide (because the coupling losses are the same for the two waveguides). In practice however, both spectra contain small periodic features due to interference of waveguide modes. For example, the presence of the $\mathrm{HE}_{12}$ mode delayed by $10 \mathrm{ps}$ with respect to the $\mathrm{HE}_{11}$ mode at the output of the $30 \mathrm{~cm}$ sample results in spectral features with periodicity of $\sim 0.1 \mathrm{THz}$ (in a region near $2.0 \mathrm{THz}$ ) [20]. A similar composition of the $\mathrm{HE}_{11}$ and $\mathrm{HE}_{12}$ modes for the $15 \mathrm{~cm}$ sample produces spectral features with periodicity of $\sim 0.2 \mathrm{THz}$. The transmission coefficient spectrum, or the ratio of the output and the input spectra,
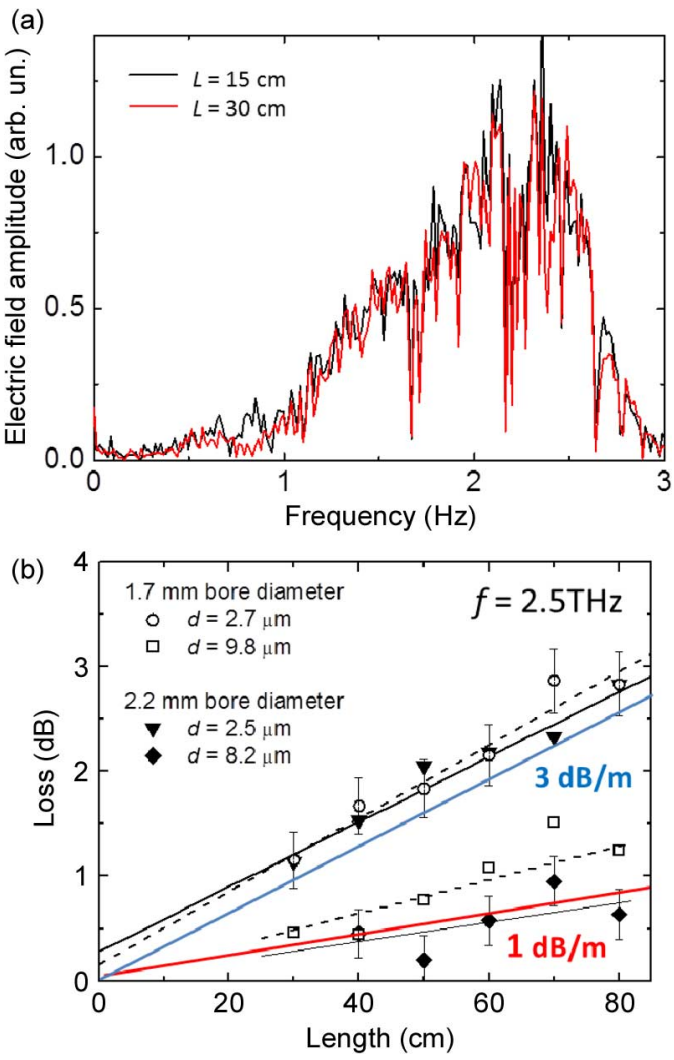

Fig. 5. (a) THz TDS spectra measured at the output of a 15 and $30 \mathrm{~cm}$ long $\mathrm{Ag} / \mathrm{PS}$ waveguides (1.8 $\mathrm{mm}$ diameter) showing periodic features due to the mode interference. Narrow absorption lines are caused by water vapour $(\sim 50 \%$ $\mathrm{RH}$ ) inside the waveguide core. (b) Single frequency cutback loss measurements at $2.54 \mathrm{THz}$ (far-infrared laser) [19]. The red and blue lines mark the transmission loss levels of $1 \mathrm{~dB} / \mathrm{m}$ and $3 \mathrm{~dB} / \mathrm{m}$ respectively.

for the test waveguide consequently contains a series of maxima and minima due to the multimode interference.

For low-loss waveguides, this systematic error can be larger than the absorption loss in the waveguide sample. The spectra for the 15 and $30 \mathrm{~cm}$ long waveguides in Fig. 5(a) are expected to differ by less than $3.5 \%$ in the electric field amplitude. The systematic error due to the mode interference however can be as large as $10 \%$ in Fig. 5(a) and it prevents achieving sufficient accuracy for the loss characterization.

The cutback loss measurement technique can be used to overcome this difficulty. The interference effects are likely to average out in a series of cutback measurements because the mode interference effects depend on the waveguide length. Fig. 5(b) shows results of cutback loss measurements performed on similar $\mathrm{THz}$ waveguides at $2.5 \mathrm{THz}$. The series of cutback measurements for six different sample lengths (up to $80 \mathrm{~cm}$ long) provides sufficient accuracy to determine the transmission loss figure of $\sim 1 \mathrm{~dB} / \mathrm{m}[19]$.

Although characterization of low-loss $\mathrm{THz}$ waveguides presents some difficulties due to multimode nature of low-loss waveguides, only experimental testing can provide reliable evaluation of transmission losses and dispersion. Developments in $\mathrm{THz}$ waveguide characterization techniques have expanded experimental research capabilities, enabled precise in-depth studies and provided a very useful capability to visualize propagating fields in $\mathrm{THz}$ waveguides. 


\section{MODELING}

The problem of wave propagation in the dielectric-lined hollow cylindrical metallic waveguide has no exact analytical solution. Several approximations however have been derived for description of the hybrid $\mathrm{HE}_{11}$ mode [33]-[35]. Here we will examine validity of two simple approximations for the mode dispersion and the mode profile in the low-loss region of the waveguide.

There are many modeling methods available to analyze the behavior of electromagnetic waveguides operating in the $\mathrm{THz}$ range of frequencies. The procedure adopted by us for the modal and dispersion analysis of the waveguides is a variational formulation in terms of the transverse magnetic field components. It is implemented using second order nodal finite elements. This formulation is derived from the Helmholtz equation for the magnetic field after eliminating the longitudinal component using the divergence-free condition [40]-[42]. This leads to the equation:

$$
\begin{array}{r}
\nabla_{t} \times\left(\varepsilon_{z z}^{-1} \nabla_{t} \times \boldsymbol{H}_{t}\right)-\hat{\mathbf{z}} \times\left[\overline{\bar{\varepsilon}}_{t t}^{-1} \cdot \nabla_{t} \times\left(\hat{\mathbf{z}} \nabla_{t} \cdot \boldsymbol{H}_{t}\right)\right]- \\
\omega^{2} \varepsilon_{0} \mu_{0} \boldsymbol{H}_{t}+\gamma^{2} \hat{\mathbf{z}} \times\left[\overline{\bar{\varepsilon}}_{t t}^{-1} \cdot\left(\hat{\mathbf{z}} \times \boldsymbol{H}_{t}\right)\right]=0
\end{array}
$$

where $\nabla_{t}=\hat{\mathbf{x}} \partial_{x}+\hat{\mathbf{y}} \partial_{y}$, the complete magnetic field is $\boldsymbol{H}=\boldsymbol{H}_{t}+\hat{\mathbf{z}} H_{z}$ and the permittivity tensor is assumed to be anisotropic and of the form: $\overline{\bar{\varepsilon}}=\overline{\bar{\varepsilon}}_{t t}+\varepsilon_{z z} \hat{\mathbf{z}} \hat{\mathbf{z}}$.

Applying a variational or a Galerkin procedure to (2) leads to a matrix eigenvalue problem of the form

$$
\left(\mathbf{A}_{1}+\omega^{2} \mathbf{A}_{2}-\gamma^{2} \mathbf{B}\right) \mathbf{h}=0
$$

where the vector $\mathbf{h}$ is formed by the nodal values of the transverse components of the magnetic field. Equation (3) can be formulated as a generalized eigenvalue problem either for the propagation constant or the frequency. The most convenient form is for the propagation constant, in particular when there are losses involved or the propagation constant is otherwise complex. In that case, (3) can be written in the form

$$
\mathbf{A h}=\gamma^{2} \mathbf{B h}
$$

where both matrices are in general complex and unsymmetric.

The main advantages of this formulation are that it is quite efficient, since it only needs two components to represent the field; and that it gives results in terms of the propagation constant that can be complex in the presence of losses. Alternative methods use either the full $\mathbf{E}$ or $\mathbf{H}$ field and are implemented with a combination of nodal and vector finite elements [43], [44].

The dielectric losses in our formulation can be taken into account by specifying the imaginary part of the dielectric function. If the metal conductivity can be considered high, the losses in the metallic walls can be estimated in a simple fashion using a perturbation approach, by determining the mode profile for the case of a perfect metal and then calculating the currents on the perfect metallic walls. The dissipated power is approximated by a contour integral of the current density $\mathrm{J}_{\mathrm{s}}$ :

$$
P_{l}=\frac{1}{2} R_{m} \oint_{C} \mathbf{J}_{\mathbf{s}} \cdot \mathbf{J}_{\mathbf{s}}^{*} d l
$$

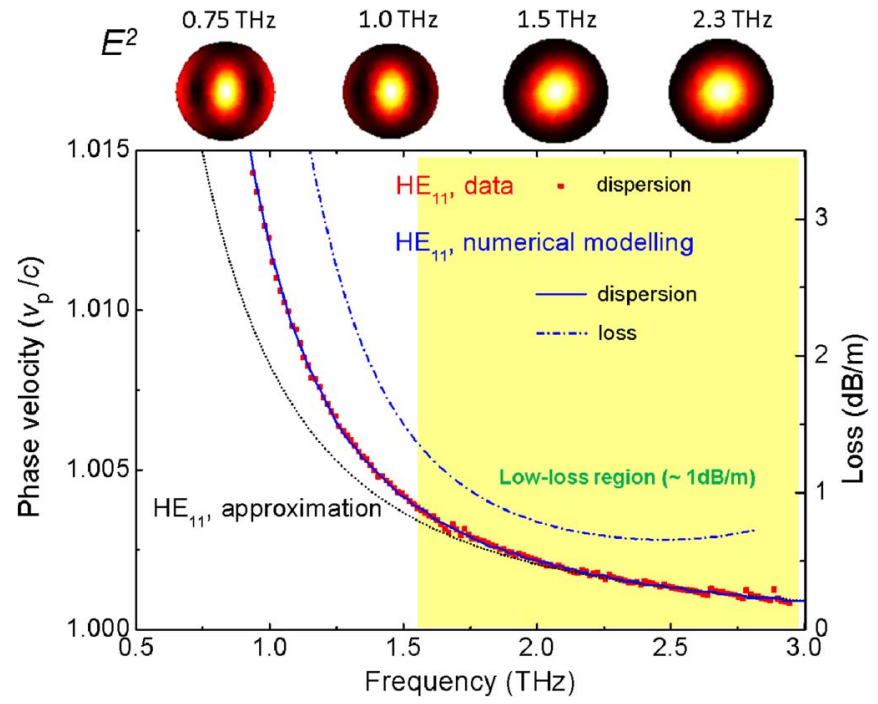

Fig. 6. Dispersion characteristics for the $\mathrm{HE}_{11}$ mode in the $1.8 \mathrm{~mm}$ diameter dielectric-lined hollow metallic waveguide. The PS coating is modelled as a 17 $\mu \mathrm{m}$ thick lossless dielectric layer. The experimental characteristics measured by THz-TDS are shown by red squares [18]. The dispersion curves are compared to the analytical approximation for the cutoff frequency of $0.128 \mathrm{THz}$. The losses in the metallic walls are estimated numerically. At low frequencies, the numerically modeled $\mathrm{HE}_{11}$ mode profiles show that significant energy density is distributed near metallic walls.

where $R_{m}$ is the surface resistance. This approach, of course, is not accurate at $\mathrm{THz}$ frequencies if the metallic losses are high. In such cases losses can be calculated by treating the metal as a non-perfect conductor in the finite element formulation of the problem. In this work, we used the perturbation approach, provided that only a small fraction of wave energy is distributed near the waveguide metallic interface.

The results of numerical calculations for the $\mathrm{HE}_{11}$ mode in the $1.8 \mathrm{~mm}$ diameter dielectric-lined waveguide are shown in Fig. 6 . The dominant mode profile agrees perfectly with the experimentally measured profile shown in Fig. 4(a). The calculated phase velocity also agrees with the dispersion data experimentally measured by THz-TDS [18]. The Ohmic losses in the waveguide are estimated according to (5). The loss spectrum is plotted on the same graph to show the spectral region where the waveguide exhibits the lowest loss of $\sim 1 \mathrm{~dB} / \mathrm{m}$. The numerical calculations show that the low-loss characteristics are achieved within a relatively large band of frequencies: for the $1.8 \mathrm{~mm}$ diameter waveguide, the low-loss region extends from $1.5 \mathrm{THz}$ to $3 \mathrm{THz}$.

The numerical results demonstrate the impact of the mode profile on the loss spectrum: the Ohmic losses are minimal only when energy of the mode is concentrated in the waveguide center, away from the absorbing walls. A series of profiles in Fig. 6 shows that a larger fraction of the mode energy is distributed near the metallic walls at low frequencies. The Ohmic losses increase correspondingly at frequencies below $1.5 \mathrm{THz}$ for this waveguide.

The dispersion curve and the mode profile can now be compared to analytical approximations in the low-loss region. The approximations are based on the assumption that the electric field vanishes at the waveguide wall and it is described by the zeroth order Bessel function [18], [35]. For the $1.8 \mathrm{~mm}$ diameter 
waveguide, this approximation yields the cutoff frequency of $0.128 \mathrm{THz}$ [18]. The analytical approximation plotted in Fig. 6 agrees well with the dispersion data within the low-loss region. The mode profile approximation is also valid in this frequency range [18]. The possibility to describe the $\mathrm{HE}_{11}$ mode by these simple analytical approximations simplifies analysis of signal transmission in the dielectric-lined hollow metallic waveguide.

\section{CONCLUSION}

Most solutions for reducing transmission losses in $\mathrm{THz}$ waveguides so far have implemented confinement of the guided wave in a large hollow or porous core. The dielectric-lined hollow cylindrical metallic waveguides are among the waveguides, which can provide the transmission loss below $1 \mathrm{~dB} / \mathrm{m}$ at $\mathrm{THz}$ frequencies. The reduced loss compared to hollow metallic waveguides is attributed to the modified profile of the dominant waveguide mode. Although the mode profile is wavelength dependent, our experimental studies and numerical calculations show that the low-loss characteristics are achieved within a relatively large band of frequencies. In fact, the dielectric-lined hollow cylindrical metallic waveguides exhibit a unique combination of low loss and relatively low dispersion. Alternative $\mathrm{THz}$ waveguides based on photonic crystal structures and porous waveguide exhibit comparable or higher losses, however they are achieved in narrower bands.

The combination of low loss and low dispersion allows using the dielectric-lined metallic waveguides not only for transmission of narrow band signals but also for transmission of $\mathrm{THz}$ pulses and we recently utilized these waveguides in a $\mathrm{THz}$ timedomain microscopy system [45]. These waveguides can also enable large-bandwidth signal transmission with carrier frequencies of 2-3 THz over distances of up to 10-30 meters (assuming the industry acceptable loss of $20 \mathrm{~dB}$ ).

Our dielectric-lined hollow metallic waveguides are fabricated by coating the inner surface of glass or polycarbonate tubing with a layer of silver and a layer of polystyrene. This process allows producing both rigid and flexible waveguides for the region of $1.5 \mathrm{THz}$ and above. The same process can be applied for lower frequency waveguides if $20-30 \mu \mathrm{m}$ thick uniform PS films can be deposited.

Further research on reduction of losses in $\mathrm{THz}$ waveguide is likely to involve development of more transparent dielectric materials for porous and photonic crystal waveguides or modifications of metallic waveguides with structured metallic/dielectric surfaces. Due to the multimode nature of most low-loss $\mathrm{THz}$ waveguides, particular attention must be given to waveguide integration with $\mathrm{THz}$ sources and detectors. It is essential to develop waveguide couplers that channel $\mathrm{THz}$ waves efficiently into the dominant waveguide mode. Mode mixing at waveguide bends and irregularities is another important area for further investigations. Although multimode waveguides are suitable for some applications, including $\mathrm{THz}$ imaging and digital communications, eliminating higher order modes by means of efficient coupling can enable quasi-single mode propagation required in other applications, e.g. THz spectroscopy.

\section{REFERENCES}

[1] G. Gallot, S. P. Jamison, R. W. McGowan, and D. Grischkowsky, "Terahertz waveguides," J. Opt. Soc. Am. B, vol. 17, pp. 851-863, 2000.

[2] S. P. Jamison, R. W. McGowan, and D. Grischkowsky, "Single-mode waveguide propagation and reshaping of sub-ps terahertz pulses in sapphire fibers," Appl. Phys. Lett., vol. 76, no. 15, pp. 1987-1989, 2000.

[3] R. Mendis and D. Grischkowsky, "Plastic ribbon THz waveguides," $J$. Appl. Phys., vol. 88, pp. 4449-4451, 2000.

[4] H. Han, H. Park, M. Cho, and J. Kim, "Terahertz pulse propagation in a plastic photonic crystal fiber," Appl. Phys. Lett., vol. 80, pp. 2634-2636, 2002.

[5] T.-I. Jeon and D. Grischkowsky, "Direct optoelectronic generation and detection of sub-ps-electrical pulses on sub-mm-coaxial transmission lines," Appl. Phys. Lett., vol. 85, pp. 6092-6094, 2004.

[6] J. A. Harrington, R. George, P. Pedersen, and E. Mueller, "Hollow polycarbonate waveguides with inner $\mathrm{Cu}$ coatings for delivery of terahertz radiation," Opt. Exp., vol. 12, pp. 5263-5268, 2004.

[7] D. Chen and H. Chen, "A novel low-loss Terahertz waveguide: Polymer tube," Opt. Exp., vol. 18, pp. 3762-3767, 2010.

[8] J.-T. Lu, Y.-C. Hsueh, Y.-R. Huang, Y.-J. Hwang, and C.-K. Sun, "Bending loss of terahertz pipe waveguides," Opt. Exp., vol. 18, p. 26332, Dec. 2010.

[9] D. Tian, H. Zhang, Q. Wen, Z. Wang, S. Li, Z. Chen, and X. Guo, "Dual cylindrical metallic grating-cladding polymer hollow waveguide for terahertz transmission with low loss," Appl. Phys. Lett., vol. 97, p. 133502, 2010.

[10] K. Nielsen, H. K. Rasmussen, A. J. Adam, P. C. Planken, O. Bang, and P. U. Jepsen, "Bendable, low-loss Topas fibers for the terahertz frequency range," Opt. Exp., vol. 17, pp. 8592-8601, 2009.

[11] C.-H. Lai, Y.-C. Hsueh, H.-W. Chen, Y. J. Huang, H. C. Chang, and C.-K. Sun, "Low-index terahertz pipe waveguides," Opt. Lett., vol. 34, pp. 3457-3459, 2009.

[12] S. Atakaramians, S. A. V. , B. M. Fischer, D. Abbot, and T. M. Monro, "Porous fibers: A novel approach to low loss THz waveguides," Opt. Exp., vol. 16, pp. 376-379, 2008.

[13] A. Hassani, A. Dupuis, and M. Skorobogatiy, "Porous polymer fibers for low-loss Terahertz guiding," Opt. Exp., vol. 16, pp. 6340-6351, 2008.

[14] T. Ito, Y. Matsuura, M. Miyagi, H. Minamide, and H. Ito, "Flexible terahertz fiber optics with low bend-induced losses," J. Opt. Soc. Am. $B$, vol. 24, pp. 1230-1235, 2007.

[15] L.-J. Chen, H.-W. Chen, T.-F. Kao, H.-Y. Lu, and C.-K. Sun, "Lowloss subwavelength plastic fiber for terahertz waveguiding," Opt. Lett., vol. 31, pp. 308-310, 2006.

[16] B. Bowden, J. A. Harrington, and O. Mitrofanov, "Silver/polystyrenecoated hollow glass waveguides for the transmission of terahertz radiation," Opt. Lett., vol. 32, pp. 2945-2947, 2007.

[17] Y. Matsuura and E. Takeda, "Hollow optical fibers loaded with an inner dielectric film for terahertz broadband spectroscopy," J. Opt. Soc. Am. $B$, vol. 25, pp. 1949-1954, 2008.

[18] O. Mitrofanov and J. A. Harrington, "Dielectric-lined cylindrical metallic THz waveguides: Mode structure and dispersion," Opt. Exp., vol. 18, pp. 1898-1903, 2010.

[19] B. Bowden, J. A. Harrington, and O. Mitrofanov, "Low-loss modes in hollow metallic terahertz waveguides with dielectric coatings," Appl. Phys. Lett., vol. 93, p. 181104, 2008.

[20] O. Mitrofanov, T. Tan, P. R. Mark, B. Bowden, and J. A. Harrington, "Waveguide mode imaging and dispersion analysis with terahertz nearfield microscopy," Appl. Phys. Lett., vol. 94, p. 171104, 2009.

[21] N. C. J. van der Valk and P. C. M. Planken, "Effect of a dielectric coating on terahertz surface plasmon polaritons on metal wires," Appl. Phys. Lett., vol. 87, p. 071106, 2005.

[22] M. Gong, T.-I. Jeon, and D. Grischkowsky, "THz surface wave collapse on coated metal surfaces," Opt. Exp., vol. 17, pp. 17088-17101, 2009.

[23] R. Mendis and D. M. Mittleman, "An investigation of the lowest-order transverse-electric $\left(\mathrm{TE}_{1}\right)$ mode of the parallel-plate waveguide for THz pulse propagation," J. Opt. Soc. Am. B, vol. 26, pp. A6-A13, 2009.

[24] T. Hidaka, H. Minamide, H. Ito, J. Nishizawa, K. Tamura, and S. Ichikawa, "Ferroelectric PVD cladding terhertz waveguide," $J$. Lightw. Technol., vol. 23, no. 8, pp. 2469-2473, 2005.

[25] M. Goto, A. Quema, H. Takahashi, S. Ono, and N. Sarukura, "Teflon photonic crystal fiber as terahertz waveguide," Jp. J. Appl. Phys., vol. 43, pp. 317-319, 2004. 
[26] M. Skorobogatiy and A. Dupuis, "Ferroelectric all-polymer hollow Bragg fibers for terahertz guidance," Appl. Phys. Lett., vol. 90, no. 11, p. $113514,2007$.

[27] Y. F. Geng, X. L. Tan, P. Wang, and J. Q. Yao, "Transmission loss and dispersion in plastic terahertz photonic band-gap fibers," Appl. Phys. $B$, vol. 91, pp. 333-336, 2008 .

[28] V. Astley, R. Mendis, and D. M. Mittleman, "Characterization of terahertz field confinement at the end of a tapered metal wire waveguide," Appl. Phys. Lett., vol. 95, p. 031104, 2009.

[29] W. Zhu, A. Agrawal, and A. Nahata, "Planar plasmonic terahertz guided-wave devices," Opt. Exp., vol. 16, pp. 6216-6226, 2008.

[30] H. Zhan, R. Mendis, and D. M. Mittleman, "Superfocusing terahertz waves below $\lambda / 250$ using plasmonic parallel-plate waveguides," Opt. Exp., vol. 18, pp. 9643-9650, 2010.

[31] J. A. Harrington, Infrared Fiber Optics and Their Applications. Bellingham, WA: SPIE Press, 2004.

[32] J. Dai, J. Zhang, W. Zhang, and D. Grischkowsky, "Terahertz time-domain spectroscopy characterization of the far-infrared absorption and index of refraction of high-resistivity, float-zone silicon," J. Opt. Soc. Am. B, vol. 21, pp. 1379-1386, 2004.

[33] J. W. Carlin and P. D'Agostino, "Normal modes in overmoded dielectric-lined circular waveguide," Bell Syst. Tech., J., vol. 52, pp. 453-486, 1973.

[34] C. Dragone, "Attenuation and radiation characteristics of the HE11 mode," IEEE Trans. Microw. Theory Tech., vol. 28, pp. 704-710, 1980.

[35] M. Miyagi and S. Kawakami, "Design theory of dielectric-coated circular metallic waveguides for infrared transmission," J. Lightw. Technol., vol. 2, pp. 116-126, 1984.

[36] X. L. Tang, Y. W. Shi, Y. Matsuura, K. Iwai, and M. Miyagi, "Tranmission characteristics of terahertz hollow fiber with an absorptive dielectric inner-coating film," Opt. Lett., vol. 34, no. 14, pp. 2231-2233, 2009.

[37] C. Themistos, B. M. A. Rahman, M. Rajarajan, K. T. V. Grattan, B. Bowden, and J. A. Harrington, "Characterization of silver/polystyrene-coated hollow glass waveguides at THz frequency," J. Lightw. Technol., vol. 25, pp. 2456-2461, 2007.

[38] B. Bowden, J. A. Harrington, and O. Mitrofanov, "Fabrication of terahertz hollow-glass metallic waveguides with inner dielectric coatings," J. Appl. Phys., vol. 104, p. 093110, 2008.

[39] M. S. Vitiello, J.-H. Xu, M. Kumar, F. Beltram, A. Tredicucci, O. Mitrofanov, H. E. Beere, and D. A. Ritchie, "High efficiency coupling of Terahertz micro-ring quantum cascade lasers to the low-loss optical modes of hollow metallic waveguides," Opt. Exp., vol. 19, pp. 1122-1130, 2011

[40] Y. Abe, Y. Matsuura, Y. W. Shi, Y. Wang, H. Uyama, and M. Miyagi, "Polymer-coated hollow fiber for $\mathrm{CO}_{2}$ laser delivery," Opt. Lett., vol. 23, no. 2, pp. 89-90, 1998.

[41] Y. Lu and F. A. Fernández, "An efficient finite element solution of inhomogeneous anisotropic and lossy dielectric waveguides," IEEE Trans. Microw. Theory Tech., vol. 41, no. 6/7, pp. 1215-1223, 1993.

[42] F. A. Fernández and Y. Lu, Microwave and Optical Waveguide Analysis by the Finite Element Method. Taunton, Somerset, U.K.: Research Studies Press, 1996.

[43] M. Koshiba, Optical Waveguide Theory by the Finite Element Method. Tokyo, Japan: KTK Scientific Publishers, 1992.

[44] J. Jin, The Finite Element Method in Electromagnetics. New York: Wiley, 2002.

[45] R. Mueckstein and O. Mitrofanov, "Imaging of terahertz surface plasmon waves excited on a gold surface by a focused beam," Opt. Exp., vol. 19, pp. 3212-3217, 2011.
Oleg Mitrofanov received the Ph.D. degree in applied physics in 2001 from the New Jersey Institute of Technology, Newark, NJ.

In 1998, he joined Bell Laboratories-Lucent Technologies, Murray Hill, NJ, as a research student to work on spectroscopy and near-field imaging with Terahertz pulses. From 2001 to 2007, he was a Member of Technical Staff at Bell Laboratories, and in 2007, he joined the academic staff of the Department of Electronic and Electrical Engineering, University College London, U.K., where he currently holds the Royal Society University Research Fellowship. His research interests are in the areas of Terahertz technology and semiconductor physics.

Richard James received the M.Eng. degree in electronic and electrical engineering from the University College London, U.K., in 2001 and the Ph.D. in 2006, supported by an EPSRC scholarship.

From 2006 to 2010, he worked as a Research Fellow in the Liquid Crystal Modeling group at University College London, on the characterization and applications of liquid crystals at microwave frequencies. In 2010, he started work at the Department of Electronics and Information Systems, Ghent University, Belgium, where he is an FWO Visiting Postdoctoral Fellow. His research interests include the numerical modeling of liquid crystal hydrodynamics and defect structures, and microwave devices with liquid crystal substrates. He is a coauthor of about 30 papers in international journals and conference proceedings.

F. Aníbal Fernández (M'88) received the B.Sc. degree in applied mathematics from the Universidad de Chile, Santiago, Chile, and the Ph.D. degree in electrical engineering from the University College London, London, U.K. in 1981.

In 1986, he joined the academic staff of the Department of Electronic and Electrical Engineering, University College London, where he has been dedicated to the development of mathematical and computer modelling methods. His research was initially applied to electromagnetic problems where he worked on methods for the analysis of microwave and optical waveguiding systems, later on nonlinear optics and, since 1994, on the modelling of liquid crystal devices. He has published one book and over 160 research papers and conference presentations in these fields.

Themistoklis K. Mavrogordatos received a Diploma in Electrical Engineering from the National Technical University of Athens, Greece, in 2009 The same year, he joined the Photonics Doctoral Training Centre (collaborative programme between the University of Cambridge and the University College London), where he is undertaking his Ph.D. project. His current research interests revolve around Fourier optics and liquid crystal lasers.

James A. Harrington received the Ph.D. degree in physics from Northwestern University, Evanston, IL, in 1970.

$\mathrm{He}$ is a Professor of material science and engineering at Rutgers University, Piscataway, NJ. He has over 30 years of research experience in the area of optical properties of solids mostly in the field of infrared fiber optics. His research interests include fabrication, characterization, and applications of specialty fiber optics for use in the delivery of laser power in surgical and industrial applications and for use as chemical and temperature fiber optic sensors. His book on Infrared Fiber Optics and Their Applications (SPIE Press, 2004) describes the many specialty fibers that transmit in the infrared.

Prof. Harrington is a Fellow of SPIE and OSA and a member of the APS. In 2005-2006 he served as a Jefferson Science Fellow at the Department of State working on export control. 